Pacific Journal of Mathematics

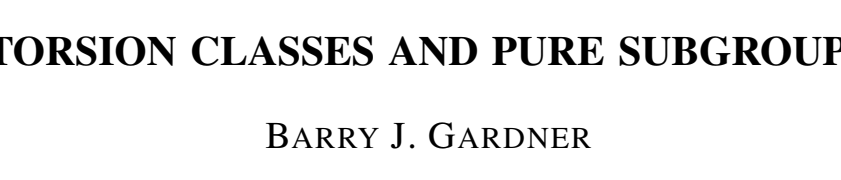




\title{
TORSION CLASSES AND PURE SUBGROUPS
}

\author{
B. J. GARDNER
}

In this note we obtain a classification of the classes $\mathscr{T}$ of abelian groups satisfying the following closure conditions: (i) If $\left\{A_{\mu} \mid \mu \in M\right\} \subseteq \mathscr{T}$, then $\mathscr{T}$ contains the direct sum $\sum A_{\mu}$.

For a short exact sequence

(*) $0 \longrightarrow A \longrightarrow B \longrightarrow C \longrightarrow 0$
(ii) $C \in \mathscr{T}$ if $B \in \mathscr{T}$
(iii) $B \in \mathscr{T}$ if $A, C \in \mathscr{T}$
(iv) $A \in \mathscr{T}$ if $B \in \mathscr{T}$ and (*) is pure.

Classes satisfying (i), (ii) and (iii) are called torsion classes (of abelian groups) and were first studied by Dickson [2], who classified those which contain only torsion groups and showed that the general classification problem reduces, essentially, to that for torsion classes determined (in the sense of $\S 2$ below) by torsion-free groups. The torsion classes which are closed under taking subgroups (called strongly-complete Serre classes) can be described quite simply ([1], [2], [10]). A possible approach to the general problem is to investigate torsion classes closed under taking the subgroups corresponding to proper classes of monomorphisms as used in relative homological algebra (see for example [8], pp. 367 et seqq.), and herein lies the motivation for the present paper.

1. Notation. "Group" means "abelian group" throughout. $h(x)$ denotes the height of an element of a torsion-free group $\tau(x)$ its type and $\tau(X)$ the type of a rational group $X$. An $S$-group, where $S$ is a set of primes, is a group whose elements have orders belonging to the multiplicative semigroup $S^{*}$ generated by $S$. A group $A$ is $p$ divisible for a prime $p$ if $p A=A$ and $S$-divisible if $p$-divisible for each $p \in S$. $\mathscr{T}_{0}, \mathscr{F}_{0}$ are the classes of all torsion and torsion-free groups respectively. For a group $A, A_{t}$ is the torsion subgroup, $A_{p}$ its $p$-primary component. The direct sum (or discrete direct sum) of a set of groups $\left\{A_{\mu} \mid \mu \in M\right\}$ is denoted by $\sum A_{\mu}$, the direct product (or complete direct sum) by $\Sigma^{*} A_{\mu}$ and an element of either by $\left(a_{\mu}\right)$. $[A, B]$ is the group of homomorphisms from a group $A$ to a group $B$. If $a$ is an element of a torsion-free group $A,[a]$ denotes the cyclic subgroup it generates, $[a]_{*}$ the smallest pure subgroup containing it. $Z$ is the group of integers, $Q$ the (additive) group of rational numbers, $Z(p)$ the cyclic group of order $p, Z\left(p^{\infty}\right)$ the quasicyclic $p$-group. For 
a set $S$ of primes, $Q(S)$ is the subgroup $\left\{m / n \mid m \in Z, n \in S^{*}\right\}$ of $Q$ and for a prime $p, Q(p)=\left\{m / p^{n} \mid m, n \in Z, n \geqq 0\right\}$. $I_{p}$ is the group or ring of $p$-adic integers.

For unexplained terms see [4].

2. Torsion classes. We begin by listing some properties of torsion classes for later use.

For a class $\mathscr{C}$ of groups we write $T(\mathscr{C})$ for the torsion class determined by $\mathscr{C}$, i.e. the smallest torsion class $\mathscr{T}$ with $\mathscr{C} \subseteq \mathscr{T}$ but if $\mathscr{C}$ has a single member $C, T(C)$ rather than $T(\{C\})$ will be used.

T1. $A \in T(\mathscr{C})$ if and only if $[A, B]=0$ whenever $[C, B]=0$ for all $C \in \mathscr{C}$. [3].

$T(\mathscr{C})$ is also the lower radical class determined by $\mathscr{C}$, in the sense of Kurosh [7]-Shul'geifer [9], so by the simplified version of the Kurosh construction which applies in an abelian category, we obtain

T2. $A \in T(\mathscr{C})$ if and only if every nonzero homomorphic image $B$ of $A$ has a nonzero subgroup which is a homomorphic image of some $C \in \mathscr{C}$, i.e., $[C, B] \neq 0$.

A torsion class $\mathscr{T}$ will be called a t-torsion class if it contains only torsion groups.

T3. Let $S_{1}, S_{2}$ be disjoint sets of primes and let $\mathscr{T}$ be the class of all groups of the form $A_{1} \oplus A_{2}$, where $A_{1}$ is an $S_{1}$-group and $A_{2} a$ divisible $S_{2}$-group. Then $\mathscr{T}$ is the t-torsion class

$$
T\left(\left\{Z(p) \mid p \in S_{1}\right\} \cup\left\{Z\left(p^{\infty}\right) \mid p \in S_{2}\right\}\right) .
$$

Any t-torsion class is uniquely represented in this way. [2].

T4. Let $\mathscr{T}$ be a torsion class and $p$ a prime. Then either $Z(p) \in \mathscr{T}$ or every group in $\mathscr{T}$ is p-divisible [2].

Proposition 2.1. If $\mathscr{T}$ is a torsion class containing a torsionfree group $A$, then $Z\left(p^{\infty}\right) \in \mathscr{T}$ for every prime $p$.

Proof. If $Z(p) \in \mathscr{T}$, then $\mathscr{T}$ contains all $p$-groups (T3); if not, then $A$ is $p$-divisible, so $\tau\left([a]_{*}\right) \geqq \tau(Q(p))$ for any nonzero $a \in A$. Thus $A /[a]$ has a subgroup and therefore a direct summand isomorphic to $Z\left(p^{\infty}\right)$, i.e. $Z\left(p^{\infty}\right)$ is a homomorphic image of $A$.

T5. A torsion class $\mathscr{T}$ contains a group $A$ if and only if $A_{t}$ 
and $A / A_{t} \in \mathscr{T}[2]$.

T6. Any torsion class $\mathscr{T}$ satisfies the equality

$$
\mathscr{T}=T\left(\left[\mathscr{T} \cap \mathscr{T}_{0}\right] \cup\left[\mathscr{T} \cap \mathscr{F}_{0}\right]\right) .
$$

[2].

T7. $T(Q(S))$ is the class of $S$-divisible groups, for any set $S$ of primes. (Cf. [2], Proposition 4.1.)

3. A simplification of the problem. As a first step, we show that every torsion class closed under taking pure subgroups is either a $t$-torsion class or is determined by rational and torsion groups. A class of the latter kind will be called an r.t.-torsion class.

Proposition 3.1. All t-torsion classes are closed under taking pure subgroups.

Proof. Let $S_{1}, S_{2}$ be disjoint sets of primes. If $A_{1}$ is an $S_{1}$-group and $A_{2}$ a divisible $S_{2}$-group, then clearly any pure subgroup of $A_{1} \oplus A_{2}$ is the direct sum of an $S_{1}$-group and a divisible $S_{2}$-group.

THEOREM 3.2. A torsion class $\mathscr{T}$ is closed under taking pure subgroups if and only if $\mathscr{T} \cap \mathscr{F}_{0}$ is.

Proof. Let $A^{\prime}$ be a pure subgroup of $A \in \mathscr{T}$, and consider the induced diagram

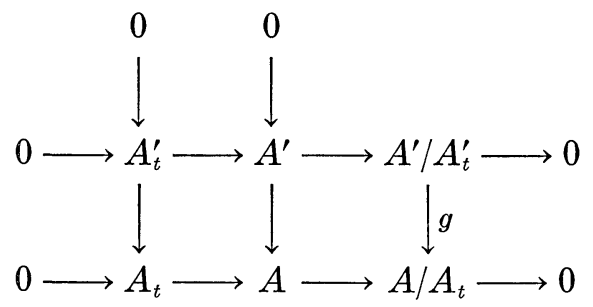

with exact rows and columns, where $g$ is defined by $g\left(a^{\prime}+A_{t}^{\prime}\right)=$ $a^{\prime}+A_{t}$. $\quad A_{t}^{\prime}$ is pure in $A^{\prime}$ and hence in $A$. Therefore $A_{t}^{\prime}$ is pure in $A_{t}$ so by Proposition 3.1, $A_{t}^{\prime} \in \mathscr{T}^{\prime} \cap \mathscr{T}_{0}$. The kernel of $g$ is $A^{\prime} \cap A_{t} / A_{t}^{\prime}=0$. If, for some nonzero $n \in Z, a^{\prime} \in A^{\prime}$ and $a \in A$ we have $g\left(a^{\prime}+A_{t}^{\prime}\right)=$ $n\left(a+A_{t}\right)$, then $m\left(a^{\prime}-n a\right)=0$ for some nonzero $m \in Z$, i.e. $m a^{\prime}=m n a$. Since $A^{\prime}$ is pure in $A$, there exists $a^{\prime \prime} \in A^{\prime}$ with $m a^{\prime}=m n a^{\prime \prime}$. But then $g\left(a^{\prime}+A_{t}^{\prime}\right)=n g\left(a^{\prime \prime}+A_{t}^{\prime}\right)$, so that $g$ is a pure monomorphism. Thus if $\mathscr{T} \cap \mathscr{F}_{0}$ is closed under taking pure subgroups, $A^{\prime} / A_{t}^{\prime} \in \mathscr{T} \cap \mathscr{F}_{0}$ 
so $A^{\prime} \in \mathscr{T}$ and $\mathscr{T}$ is therefore closed under taking pure subgroups. The converse is obvious.

THEOREM 3.3. If a torsion class $\mathscr{T}$ is closed under taking pure subgroups, then

$$
\mathscr{T}=T\left(\left[\mathscr{T} \cap \mathscr{T}_{0}\right] \cup \overline{\mathscr{T}}\right)
$$

where $\overline{\mathscr{T}}$ is the class of rational groups in $\mathscr{T}$.

The proof uses the following lemmas:

Lemma 3.4. For $\mathscr{T}$ and $\overline{\mathscr{T}}$ as in Theorem 3.3, $\mathscr{T} \cap \mathscr{F}_{0}=$ $T(\overline{\mathscr{T}}) \cap \mathscr{F}_{0}$.

Proof. Clearly $\mathscr{T} \cap \mathscr{F}_{0} \supseteqq T(\overline{\mathscr{T}}) \cap \mathscr{F}_{0}$. Let $A$ be any group in $\mathscr{I} \cap \mathscr{F}_{0}$. Then $A$ is a homomorphic image of $\Sigma[a]_{*}$ where the sum extends over all $a \in A$ and each $[a]_{*} \in \mathscr{T}$, so $A \in T(\overline{\mathscr{T}})$.

Lemma 3.5. For any two classes $\mathscr{C}_{1}, \mathscr{C}_{2}$ of groups, $T\left(\mathscr{C}_{1} \cup \mathscr{C}_{2}\right)=$ $T\left(T\left[\mathscr{C}_{1}\right] \cup T\left[\mathscr{C}_{2}\right]\right)$.

To complete the proof of Theorem 3.3, we observe that

$$
\begin{aligned}
\mathscr{T} & =T\left(\left[\mathscr{T} \cap \mathscr{T}_{0}\right] \cup\left[\mathscr{T} \cap \mathscr{F}_{0}\right]\right) \\
& \cong T\left(\left[\mathscr{T} \cap \mathscr{T}_{0}\right] \cup T(\overline{\mathscr{T}})\right) \quad=T\left(\left[\mathscr{T} \cap \mathscr{T}_{0}\right] \cup\left[T(\overline{\mathscr{T}}) \cap \mathscr{F}_{0}\right] \cup \overline{\mathscr{T}}\right) \leqq \mathscr{T} .
\end{aligned}
$$

We conclude this section by showing that not every r.t. torsion class is closed under taking pure subgroups.

Proposition 3.6. Let $\mathscr{T}$ be a torsion class closed under taking pure subgroups and $\Gamma$ the set of types of rational groups in $\mathscr{T}$. If $\gamma, \delta \in \Gamma$, then $\gamma \cap \delta \in \Gamma$.

Proof. Let $X$ and $Y$ be rational groups with $\tau(X)=\gamma$ and $\tau(Y)=\delta$. Then $X \oplus Y$ has elements and therefore pure rational subgroups of type $\gamma \cap \delta$.

Thus for example if $p$ and $q$ are distinct primes, $T(\{Q(p), Q(q)\})$ is not closed under taking pure subgroups since $\tau(Q(p)) \cap \tau(Q(q))=\tau(Z)$ and $[Q(p), Z]=0=[Q(q), Z]$.

4. The main results. In this section we obtain an explicit characterization of the torsion classes closed under taking pure subgroups.

Lemma 4.1. Let $X$ be a rational group and $S=\{p$ prime $\mid X$ is 
p-divisible\}. Then $I_{p} \in T(X)$ whenever $p \notin S$.

Proof. Let $P$ be the set of primes distinct from $p$. Then $I_{p} \in T(Q(P))(\mathrm{T} 7)$. Also, there is a short exact sequence

$$
0 \longrightarrow X \longrightarrow Q(P) \longrightarrow \sum Z\left(q^{\infty}\right) \longrightarrow 0
$$

where $q$ ranges over $P-S$. Since $\sum Z\left(q^{\infty}\right) \in T(X)$ (Proposition 2.1), it follows that $T(X)$ contains $Q(P)$ and hence $I_{p}$.

The main result can now be stated.

Theorem 4.2. A torsion class $\mathscr{T}$ is closed under taking pure subgroups if and only if either

(i) $\mathscr{T}$ is a t-torsion class

or (ii) $\mathscr{T}=T(\{Z(p) \mid p \in P\} \cup\{Q(S)\})$, where $P$ and $S$ are sets of primes with $P \subseteq S$.

The proof of Theorem 4.2 will be accomplished in several stages. We first prove

LEMmA 4.3. Let $\left\{X_{\mu} \mid \mu \in M\right\}$ be a set of rational groups. Let $A=\sum X_{\mu}$ and $S=\{p$ prime $\mid A$ is p-divisible $\}$. Then $T\left(\left\{X_{\mu} \mid \mu \in M\right\}\right)$ contains $\Sigma^{*} A_{i}, i=1,2,3, \cdots$, where each $A_{i}=A$.

Proof. Let $f: \Sigma * A_{i} \rightarrow Y$ be a nonzero epimorphism. We show that $\left[X_{\mu}, Y\right] \neq 0$ for at least one value of $\mu$.

If $Y_{p} \neq 0$ for some $p$, then since $Y$ is $S$-divisible, so is $Y_{p}$. If $p \in S, Y_{p}$ is therefore a direct sum of copies of $Z\left(p^{\infty}\right)$ so by Proposition 2.1, $Y_{p} \in T\left(X_{\mu}\right)$ for each $\mu$ and a fortiori $\left[X_{\mu}, Y\right] \neq 0$ for all $\mu$. If $p \notin S$, then at least one $X_{\mu}$ is $p$-reduced, whence $\left[X_{\mu}, Y_{p}\right] \neq 0$.

If $Y$ is torsion-free, there are two possibilities. If $f\left(\left(a_{i}\right)\right) \neq 0$ for some $\left(a_{i}\right)$ with almost all $a_{i}=0$, then $f$ induces a nonzero map from some $A_{i}$, and hence from some $X_{\mu}$, into $Y$, while if $f\left(\left(a_{i}\right)\right)=0$ whenever $a_{i}=0$ for almost all values of $i$, then $f$ factorizes as

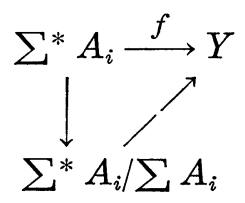

where the other maps are epimorphisms. $\sum^{*} A_{i} / \sum A_{i}$ is algebraically compact (see [6]), and also torsion-free, since $\sum A_{i}$ is a pure subgroup of $\sum^{*} A_{i}$. Thus $\sum^{*} A_{i} / \sum A_{i}$ is the direct sum of a divisible group and a (reduced) cotorsion group [5]; so, therefore, is $Y$, which being torsion- 
free is algebraically compact [5]. Since $Y$ is $S$-divisible, it has the form $D \oplus \Sigma^{*} R_{p}, p \notin S$ where each $R_{p}$ is inter alia a reduced $I_{p}$-module and $D$ is divisible. If $D \neq 0$ then for each $\mu \in M$ there are monomorphisms $X_{\mu} \rightarrow Q \rightarrow D$. If $D=0$, let $R_{p} \neq 0$. Then at least one $X_{\mu}$ is $p$-reduced, so by Lemma 4.1, $I_{p} \in T\left(X_{\mu}\right)$. Since there is an epimorphism (an $I_{p}$-epimorphism) from a direct sum of copies of $I_{p}$ to $R_{p}$, we have $R_{p} \in T\left(I_{p}\right) \subseteq T\left(X_{\mu}\right)$, so $\left[X_{\mu}, R_{p}\right] \neq 0$ and the proof is complete.

The next step is to show when $T\left(\left\{X_{\mu} \mid \mu \in M\right\}\right)$ is closed under taking pure subgroups.

LEMMA 4.4. With the notation of Lemma 4.3, if $T\left(\left\{X_{\mu} \mid \mu \in M\right\}\right)$ is closed under taking pure subgroups, it contains $Q(S)$.

Proof. Let $p_{1}, p_{2}, p_{3}, \cdots$ be the natural enumeration of the primes, and let $J=\left\{i \mid p_{i} \notin S\right\}$. For each $j \in J$, choose $a_{j} \in A$ with $h_{j}\left(a_{j}\right)=0$, where $h_{j}$ denotes height at $p_{j}$. For example, let $a_{j}=\left(x_{j \mu}\right)$ with $x_{j \mu} \in X_{\mu}$ satisfying the following conditions: (i) $x_{j \lambda} \neq 0$ for some $\lambda \in M$ for which $X_{\lambda}$ is $p_{j}$-reduced; (ii) $h_{j}\left(x_{j \lambda}\right)=0$; (iii) $x_{j \mu}=0$ for $\mu \neq \lambda$. For a natural number $i \notin J$, let $a_{i}$ be an arbitrary element of $A$, and regard the resulting $\left(a_{i}\right)$ as an element of a group $\Sigma^{*} A_{i}, i=1,2,3, \cdots$. Then $h\left(\left(a_{i}\right)\right)=\bigcap_{i=1}^{\infty} h\left(a_{i}\right)$. In particular, $h_{j}\left(\left(a_{i}\right)\right)=0$. Therefore, since $\Sigma^{*} A_{i}$ is $S$-divisible, the height of $\left(a_{i}\right)$ at a prime $p$ is infinite if $p \in S$ and zero otherwise, i.e., $\tau\left(\left(a_{i}\right)\right)=\tau(Q(S))$ and $\Sigma^{*} A_{i}$ has a pure subgroup isomorphic to $Q(S)$. By Lemma 4.3 and assumption, therefore, $Q(S) \in T\left(\left\{X_{\mu} \mid \mu \in M\right\}\right)$.

Since each $X_{\mu}$ is $S$-divisible and $T(Q(S))$ is the class of all $S$ divisible groups (T7) we have

Corollary 4.5. With the notation of Lemma 4.3, if $T\left(\left\{X_{\mu} \mid \mu \in M\right\}\right)$ is closed under taking pure subgroups, it is the class of all S-divisible groups.

Proof of Theorem 4.2. Let $\mathscr{T}$ be a torsion class closed under taking pure subgroups. If $\mathscr{T}^{-}$is not a $t$-torsion class, let $\Gamma$ be the set of types of rational groups in $\mathscr{T}$ and for each $\gamma \in \Gamma$ let $X_{r}$ be a rational group of type $\gamma$. Then

$$
\begin{aligned}
\mathscr{T} & =T\left(\left[\mathscr{T} \cap \mathscr{T}_{0}\right] \cup\left\{X_{\gamma} \mid \gamma \in \Gamma\right\}\right) & & \text { (Theorem 3.3) } \\
\text { and } \mathscr{T} \cap \mathscr{F}_{0} & =T\left(\left\{X_{\gamma} \mid \gamma \in \Gamma\right\}\right) \cap \mathscr{F}_{0} & & \text { (Lemma 3.4). }
\end{aligned}
$$

By Theorem 3.2, $T\left(\left\{X_{r} \mid \gamma \in \Gamma\right\}\right)$ is closed under taking pure subgroups and therefore, by Corollary 4.5 , is the class of all $S$-divisible groups, where $S$ is the set of all primes dividing $\sum X_{r}$. Thus $\mathscr{T}=$ 
$T\left(\left[\mathscr{T} \cap \mathscr{T}_{0}\right] \cup\{Q(S)\}\right)$. Let $P=\{p \in S \mid Z(p) \in \mathscr{T}\}$. Since $T(Q(S)) \subseteq$ $\mathscr{T}, \mathscr{T}$ contains the groups $Z\left(p^{\infty}\right)$ for all primes $p$ as well as $Z(p)$ for primes $p \notin S$. Thus by T3 and Lemma 3.5

$$
\begin{aligned}
\mathscr{T} & =T\left(\{Z(p) \mid p \notin S\} \cup\{Z(p) \mid p \in P\} \cup\left\{Z\left(p^{\infty}\right) \mid \text { all } p\right\} \cup\{Q(S)\}\right) \\
& =T(\{Z(p) \mid p \in P\} \cup\{Q(S)\}) .
\end{aligned}
$$

Conversely, that any class $\mathscr{T}=T(\{Z(p) \mid p \in P\} \cup\{Q(S)\})$ with $P \subseteq S$ is closed under taking pure subgroups follows from Theorem 3.2, Lemma 3.4 and the observation that $T(Q(S))$ is closed under taking pure subgroups. By Proposition 3.1, the proof is now complete.

Note that by $\mathrm{T} 1$, for a torsion class $\mathscr{T}$ which is not a $t$-torsion class, the representation $\mathscr{T}=T(\{Z(p) \mid p \in P\} \cup\{Q(S)\})$ is unique. We conclude with a characterization of the groups in such a class:

Proposition 4.6. A group A belongs to $\mathscr{T}=T(\{Z(p) \mid p \in P\} \cup\{Q(S)\})$ where $P$ and $S$ are sets of primes with $P \subseteq S$, if and only if there is a short exact sequence

$$
0 \longrightarrow A^{\prime} \longrightarrow A \longrightarrow A^{\prime \prime} \longrightarrow 0
$$

where $A^{\prime}$ is a P-group and $A^{\prime \prime}$ is S-divisible.

Proof. Let $A \in \mathscr{T}$ and $A^{\prime}=\sum A_{p}$ where the sum extends over all $p \in P, A^{\prime \prime}=A / A^{\prime}$. Then $A_{t}^{\prime \prime}$ has no $P$-component and belongs to $\mathscr{T}$ (T5) so therefore has divisible $S$-component. Thus $A_{t}^{\prime \prime}$ is $S$-divisible. $A^{\prime \prime} \mid A_{t}^{\prime \prime}$ is torsion-free and belongs to $\mathscr{T}$. If not $S$-divisible, it has a nonzero $S$-reduced torsion free homomorphic image $B$. But then $B \in \mathscr{T}$ and $[Q(S), B]=0=[Z(p), B]$ for each $p \in P$ and this contradicts T1, so $A^{\prime \prime} \mid A_{t}^{\prime \prime}$ is $S$-divisible, whence $A^{\prime \prime}$ is also. The converse is obvious.

\section{REFERENCES}

1. S. Balcerzyk, On classes of abelian groups, Fund. Math. 51 (1962), 149-178.

2. S. E. Dickson, On torsion classes of abelian groups, J. Math. Soc. Japan 17 (1965), $30-35$.

3. - A torsion theory for abelian categories, Trans. Amer. Math. Soc. 121 (1966), 223-235.

4. L. Fuchs, Abelian groups, Budapest, 1958.

$5 . \quad$ Notes on abelian groups II, Acta Math. Acad. Sci. Hung. 11 (1960), 117125.

6. A. Hulanicki, The structure of the factor group of the unrestricted sum by the restricted sum of abelian groups, Bull. Acad. Polon. Sci. Sér. Sci. Math. Astron. Phys. 10 (1962), 77-80.

7. A. G. Kurosh, Radicals in rings and algebras, Mat. Sb. 33 (1953), 13-26 (Russian).

8. S. MacLane, Homology, Berlin, Springer, 1963.

9. E. G. Shul'geifer, On the general theory of radicals in categories, Mat. Sb. 51 (1960), 
487-500 (Russian). English translation: Amer. Math. Soc. Trans. (Second Series) 59, 150-162.

10. E. A. Walker, Quotient categories and quasi-isomorphisms of abelian groups, Proceedings of the Colloquium on Abelian Groups, Tihany, 1963, 147-162.

Received July 2, 1969.

The University of Tasmania

Hobart, Tasmania, Australia 


\section{PACIFIC JOURNAL OF MATHEMATICS}

\section{EDITORS}

H. SAMELSON

Stanford University

Stanford, California 94305

\section{RichaRd PIERCe}

University of Washington

Seattle, Washington 98105
J. DUGUNDJI

Department of Mathematics

University of Southern California

Los Angeles, California 90007

RICHARD ARENS

University of California

Los Angeles, California 90024

\section{ASSOCIATE EDITORS}

E. F. BECKENBACH

B. H. NeUManN

F. WOLF

K. YosHIDA

\section{SUPPORTING INSTITUTIONS}

UNIVERSITY OF BRITISH COLUMBIA CALIFORNIA INSTITUTE OF TECHNOLOGY UNIVERSITY OF CALIFORNIA MONTANA STATE UNIVERSITY

UNIVERSITY OF NEVADA

NEW MEXICO STATE UNIVERSITY

OREGON STATE UNIVERSITY

UNIVERSITY OF OREGON

OSAKA UNIVERSITY

UNIVERSITY OF SOUTHERN CALIFORNIA
STANFORD UNIVERSITY

UNIVERSITY OF TOKYO

UNIVERSITY OF UTAH

WASHINGTON STATE UNIVERSITY

UNIVERSITY OF WASHINGTON

${ }^{*} \quad{ }^{*} \quad{ }^{*}$
AMERICAN MATHEMATICAL SOCIETY
CHEVRON RESEARCH CORPORATION
TRW SYSTEMS
NAVAL WEAPONS CENTER




\section{Pacific Journal of Mathematics}

\section{Vol. 33, No. $1 \quad$ March, 1970}

Mir Maswood Ali, On some extremal simplexes ................... 1

Silvio Aurora, On normed rings with monotone multiplication........... 15

Silvio Aurora, Normed fields which extend normed rings of integers....... 21

John Kelly Beem, Indefinite Minkowski spaces..................... 29

T. F. Bridgland, Trajectory integrals of set valued functions ........... 43

Robert Jay Buck, A generalized Hausdorff dimension for functions and sets ......................................... 69

Vlastimil B. Dlab, A characterization of perfect rings . . . . . . . . . . . . 79

Edward Richard Fadell, Some examples in fixed point theory ............ 89

Michael Benton Freeman, Tangential Cauchy-Riemann equations and uniform approximation ............................. 101

Barry J. Gardner, Torsion classes and pure subgroups ................ 109

Vinod B. Goyal, Bounds for the solution of a certain class of nonlinear

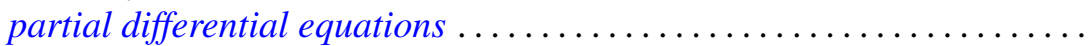

Fu Cheng Hsiang, On C, 1 summability factors of Fourier series at a given

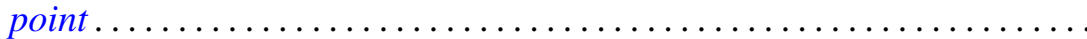

Lawrence Stanislaus Husch, Jr., Homotopy groups of PL-embedding

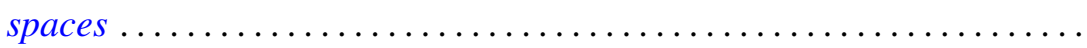

Daniel Ralph Lewis, Integration with respect to vector measures..........

Marion-Josephine Lim, $\mathscr{L}-2$ subspaces of Grassmann product spaces

Stephen J. Pierce, Orthogonal groups of positive definite multilinear functionals

W. J. Pugh and S. M. Shah, On the growth of entire functions of bounded index.

Siddani Bhaskara Rao and Ayyagari Ramachandra Rao, Existence of triconnected graphs with prescribed degrees . . .

Ralph Tyrrell Rockafellar, On the maximal monotonicity of subdifferential

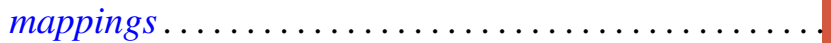

R. Shantaram, Convergence of a sequence of transformations of distribution functions. II ...............................

Julianne Souchek, Rings of analytic functions..............

Ted Joe Suffridge, The principle of subordination applied to functions of several variables...

Wei-lung Ting, On secondary characteristic classes in cobordism

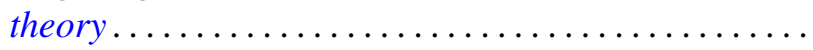

Pak-Ken Wong, Continuous complementors on $B^{*}$-algebras ...

Miyuki Yamada, On a regular semigroup in which the idempotents form a band. 